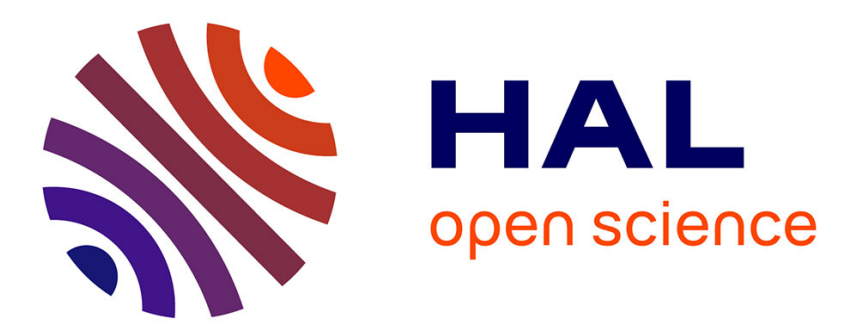

\title{
Accumulation potentielle de matière sèche et d'azote chez le lupin blanc de printemps (Lupinus albus L.)
}

Claude Duthion, Noëlle Amarger, André A. Mariotti, Vincent Durey, Jean-Jacques Giraud, Josette Gonthier, Patrick Mathey, Georges Sommer

\section{To cite this version:}

Claude Duthion, Noëlle Amarger, André A. Mariotti, Vincent Durey, Jean-Jacques Giraud, et al.. Accumulation potentielle de matière sèche et d'azote chez le lupin blanc de printemps (Lupinus albus L.). Agronomie, 1987, 7 (8), pp.585-593. hal-00885030

\section{HAL Id: hal-00885030 https://hal.science/hal-00885030}

Submitted on 1 Jan 1987

HAL is a multi-disciplinary open access archive for the deposit and dissemination of scientific research documents, whether they are published or not. The documents may come from teaching and research institutions in France or abroad, or from public or private research centers.
L'archive ouverte pluridisciplinaire HAL, est destinée au dépôt et à la diffusion de documents scientifiques de niveau recherche, publiés ou non, émanant des établissements d'enseignement et de recherche français ou étrangers, des laboratoires publics ou privés. 


\title{
Accumulation potentielle de matière sèche et d'azote chez le lupin blanc de printemps (Lupi- nus albus L.)
}

\author{
Claude DUTHION, Noëlle AMARGER*, André MARIOTTI** \\ Georges SOMMER* \\ I.N.R.A., Laboratoire d'Agronomie, \\ * Lahoratoire de Microbiologie des Sols, 17, rue Sullv, F 21034 Diion Ceder. \\ ** I.N.R.A., Laboratoire de Biogéochimie des isotopes stables, \\ Université Pierre et Marie Curie, 4, place Jussieu, F 75252 Paris Cedex 05
}

avec la collaboration technique de Vincent Durey, Jean-Jacques Giraud*, Josette Gonthier, Patrick Mathfy,

RÉSUMÉ

En vue d'acquérir les premières références sur le comportement potentiel de l'espèce en culture de printemps, le lupin blanc (cv Kali) a été cultivé durant les 3 années 1979 à 1981 sur le domaine expérimental I.N.R.A. de Bretenière (21). Les plantes ont été inoculées au semis par Rhizobium lupini et leur alimentation hydrique n'a pas été limitante. La croissance pondérale, l'accumulation de l'azote, la répartition de la matière sèche (MS) et de l'azote ont été suivies pendant le cycle. Les résultats sont donnés dans une représentation du temps en sommes de températures.

L'évolution de l'élaboration de la MS et celle de l'accumulation de $\mathrm{N}$ par les parties aériennes montrent des similitudes ; en particulier la période de forte accumulation de $\mathrm{N}\left(2,5 \mathrm{~kg} / \mathrm{ha} / 10^{\circ} \mathrm{C}-\mathrm{j}\right)$ cö̈ncide sensiblement avec la période de forte croissance pondérale $\left(80\right.$ à $\left.110 \mathrm{~kg} \mathrm{MS} / \mathrm{ha} / 10^{\circ} \mathrm{C}-\mathrm{j}\right)$. Pendant la mise en place de l'appareil reproducteur, la croissance végétative est importante ; l'appareil végétatif acquiert environ $2 / 3$ de sa MS et de son $N$ pendant la floraison, à la fin de laquelle il atteint poids et $\mathrm{N}$ accumulé maximaux. Au-delà de cette date, tous les assimilats élaborés et les composés mobilisés aux dépens des organes sénescents sont dirigés vers l'appareil reproducteur. Les transferts vers les graines se poursuivent tardivement. Les répartitions de la MS et de N sont différentes ; les indices de récolte, mesurés à maturité, sont respectivement d'environ 0,4 pour la MS et 0,8 pour $\mathrm{N}$. Les mobilisations de $\mathrm{N}$ sont de $8 \mathrm{~kg}$ environ pour $100 \mathrm{~kg}$ de graines récoltées.

Le cv Kali a élaboré $10 \mathrm{t} / \mathrm{ha}$ de MS, pour un rendement maximal de $4 \mathrm{t} / \mathrm{ha}$ de graines, et accumulé $300 \mathrm{~kg} / \mathrm{ha}$ d'azote ; ces quantités sont proches des potentiels des cv précoces actuels.

La fixation d'azote atmosphérique débute peu avant la phase de pleine croissance et s'achève au moment de la chute des dernières feuilles. La quantité de $\mathrm{N}$ fixé, mesurée en 1981 , a représenté 80 p. 100 du $\mathrm{N}$ accumulé, attei gnant $234 \mathrm{~kg} /$ ha en fin de végétation. Un bilan fait à partir des données de 1981 a montré que $47 \mathrm{p} .100$ du $\mathrm{N}$ des graines provenaient d'incorporations pendant la phase de remplissage des graines et $53 \mathrm{p} .100$ de redistributions à partir de $\mathrm{N}$ accumulé avant cette phase.

La comparaison des données présentées avec les résultats connus pour le soja, dont le lupin blanc est un possible substitut, a montré que les potentiels de production des 2 espèces sont voisins.

Mots clés additionnels : Répartition MS, répartition $N$, fixation symbiotique.

In order to obtain indications on potential canopy behaviour, spring white lupin, cv. Kali, was cultivated during the 3 years 1979-1980-1981 at Bretenière (Côte-d'Or), on the I.N.R.A. experimental farm. Plants were inoculated with Rhizobium lupini at sowing ; water was not a limiting factor. Weight growth, $\mathrm{N}$ accumulation, dry matter and $\mathrm{N}$ distribution were followed during plant life. For presentation of results, time is expressed in sums of temperatures. Dry matter production and $\mathrm{N}$ accumulation showed similar courses ; particularly, period for the greatest $\mathrm{N}$ accumulation $\left(2.5 \mathrm{~kg} / \mathrm{ha} / 10^{\circ} \mathrm{C}-\mathrm{d}\right)$ coincided approximately with the time of rapid growth $(80$ to $110 \mathrm{~kg} / \mathrm{ha} / 10^{\circ} \mathrm{C}$-d). During the setting up of the reproductive system, vegetative growth was large ; vegetative plant parts obtained about $2 / 3$ of their DM and $\mathrm{N}$ during flowering and were maximal at the end of this period. Thereafter all assimilates and compounds mobilized from senescent organs were sent to the reproductive system. Transfers to the seedfill continued late. Distributions of DM and $\mathrm{N}$ were different; harvest indices were about 0.4 for DM and 0.8 for N. N mobilization was about $80 \mathrm{~kg}$ per t seeds. Cultivar Kali produced $10 \mathrm{t} / \mathrm{ha}$ DM, with $4 \mathrm{t} / \mathrm{ha}$ of seeds as maximal yield, and accumulated $300 \mathrm{~kg} / \mathrm{ha}$ nitrogen ; these amounts are near the potential production of present early cultivars. Nitrogen fixation began just before the rapid growth period and ceased when the uppermost leaves were falling. Amount of fixed $\mathrm{N}$, which was measured in 1981, was $80 \%$ of accumulated $\mathrm{N}$, reaching $234 \mathrm{~kg} / \mathrm{ha}$. A balance sheet from data of this year showed that $47 \%$ of $\mathrm{N}$ in seeds came by assimilation during the seedfilling period and $53 \%$ by redistribution of $\mathrm{N}$ accumulated before this time. 
Comparison of the data of this study with known results about soybean, for which white lupin is a substitute, have shown that the potential production of the 2 species is approximately equal.

Additional key words : $D M$ distribution, $N$ distribution, $N$ symbiotic fixation.

\section{INTRODUCTION}

Depuis que GLADSTONES (1970) a attiré l'attention sur les possibilités d'introduction en culture de plusieurs espèces du genre Lupinus, dont on sait sélectionner des cultivars sans alcaloïdes, l'intérêt agricole du lupin blanc (Lupinus albus L.) est à nouveau amplement reconnu (LACONDE, 1985; LENOBLE, 1984). Son potentiel de rendement est élevé, sa graine, riche en protéines et en huile, en fait un excellent substitut au soja (Glycine max (L.) Merr.) dans l'alimentation animale. Cependant les surfaces qu'il occupe sont encore faibles et ce ne sont pas seulement des obstacles économiques qui freinent leur extension. Pour des raisons mal déterminées, mauvaise application des techniques disponibles ou méconnaissance de la physiologie de la plante, les rendements réellement obtenus sont en effet d'une grande variabilité, souvent très inférieurs aux rendements espérés.

Les données venant du champ, qui pourraient avoir valeur de références concernant le développement, la croissance, les besoins du lupin blanc en conditions de peuplement, sont peu nombreuses. VAVILOV \& GATAULINA (1982) ont présenté des données moyennes pour le cycle d'un cultivar précoce et établi quelques liaisons entre le rendement et des caractéristiques du couvert végétal. GATAULINA \& BEREZHNAYA (1971) ont fourni des indications sur la dynamique d'accumulation des éléments majeurs et leur répartition en fin de culture. AMARGER \& DUTHION (1983), étudiant le rôle des 2 modes de nutrition azotée, ont montré que la fixation symbiotique de l'azote atmosphérique permet les meilleurs rendements quantitatif et qualitatif.

Dans les essais que nous avons conduits (AMARGER \& DuthION, 1983), l'élaboration de la matière sèche aérienne (MS), l'accumulation d'azote par le lupin blanc, leur distribution entre les différents organes ont été suivies ; leur évolution a été notée par rapport aux phases successives du cycle. Les mesures des activités fixatrices de $\mathrm{N}$ atmosphérique et des quantités de $\mathrm{N}$ fixé ont complété ces observations. Les résultats des 3 années expérimentales correspondant à l'un des traitements imposés, pour lequel les conditions de nutrition hydrique et azotée n'ont pas été limitantes, font l'objet de cet article. Leur présentation a pour objectif de proposer des références concernant l'accumulation potentielle de MS et de $\mathrm{N}$ par la culture et de fournir une première approche des règles d'affectation de l'une et de l'autre, traduites globalement en fin de végétation par des indices de récolte. Leur comparaison avec les résultats connus pour le soja devrait permettre de mieux situer les possibilités de l'espèce.

\section{MATÉRIELS ET MÉTHODES}

Les résultats présentés proviennent de dispositifs expérimentaux de type factoriel, avec 4 répétitions en blocs, implantés sur le domaine I.N.R.A. d'Epoisses (Bretenière, Côte-d'Or), durant 3 années consécutives de 1979 à 1981. Les 2 facteurs combinés dans ces essais sont l'inoculation par Rhizobium lupini (oui/non) et la fumure azotée minérale à 4 niveaux $(0,40,80$, $160 \mathrm{~kg} / \mathrm{ha}$ ) apportée, sous forme d'ammonitrate, 1 semaine après le semis. Pour cet article, les résultats relatifs au traitement inoculation sans apport d'azote ont seuls été retenus. A celui-ci correspondent en effet les rendements et teneurs en $\mathrm{N}$ des graines maximaux ; il est en outre conforme aux conditions habituelles de culture des légumineuses dont l'alimentation azotée est assurée par la symbiose avec Rhizobium et la fourniture du sol. Le cultivar (cv) de printemps précoce Kali, d'origine polonaise, a été utilisé.

La notation adoptée, dans le texte qui suit, pour désigner les différents éléments de la structure du lupin blanc est conforme aux suggestions de FARRINGTON \& GREENWOOD (1975). La plante est constituée d'un axe principal $\mathrm{A} 0$ et de ramifications successives, émises à l'aisselle des feuilles les plus élevées du ou des axes de l'ordre immédiatement inférieur. On aura ainsi des axes apicaux d'ordre 1 puis 2 , puis 3 notés A1, A2, A3. Chaque axe est susceptible de porter une inflorescence terminale.

\section{A. Conditions générales des essais}

La conduite des essais et les dates des principales interventions (AMARGER \& DUTHION, 1983) ne sont pas rappelées.

\section{Les peuplements}

Les semis ont été réalisés à l'aide d'un semoir pneumatique, aux densités de 80 à 100 graines $/ \mathrm{m}^{2}$ préconisées par BERTHAUT \& SIXDENIER (1978) pour la région Centre-Est. Les densités moyennes de peuplement à la récolte, évaluées à partir de 6 dénombrements sur un mètre linéaire par parcelle élémentaire, ont été de 92 , 68 et 72 plantes $/ \mathrm{m}^{2}$ en 1979,1980 et 1981 . Une attaque de Botrytis cinerea, survenant en fin de floraison en 1980, a entraîné quelques disparitions de plantes et des destructions de gousses; aucun comptage n'en a été fait.

\section{Le sol}

Le sol est un sol brun calcique argileux, reposant sur un cailloutis alluvial calcaire situé à une profondeur moyenne de 50 à $70 \mathrm{~cm}$ selon les parcelles. Sa teneur en 
azote total est voisine de 0,2 p. 100. Assez bien pourvu en acide phosphorique et potasse, il a reçu 120 unités de l'un et l'autre avant chaque culture.

Les parcelles expérimentales n'ont jamais porté de lupin. Le nombre de $R$. lupini présents avant semis est de 10 à 50,3 à 30 et inférieur à 0,1 pour $100 \mathrm{~g}$ de sol, respectivement en 1979,1980 et 1981 . Ce nombre est très inférieur au seuil de $10^{3}$ bactéries jugé convenable pour une bonne nodulation.

\section{Les conditions climatiques et les bilans hydriques}

Les températures moyennes mensuelles et les durées d'insolation durant les 3 saisons de culture sont présentées dans le tableau 1.

\section{TABLEAU 1}

Conditions climatiques pendant les 3 années d'expérimentation. Climatic conditions during experiments.

\begin{tabular}{|c|c|c|c|c|c|}
\hline & A & M & $J$ & $\mathbf{J}$ & A \\
\hline \multicolumn{6}{|l|}{$\begin{array}{l}\text { Température moyenne } \\
\text { mensuelle }\left({ }^{\circ} \mathrm{C}\right)\end{array}$} \\
\hline 1979 & 8,0 & 12,7 & 16,8 & 18,4 & 16,5 \\
\hline 1980 & 7,7 & 11,7 & 15,2 & 16,5 & 18,9 \\
\hline 1981 & 10,1 & 12,2 & 15,4 & 17,8 & 18,4 \\
\hline Moyenne $1961 / 1980$ & 8,8 & 12,9 & 16,2 & 18,4 & 18,0 \\
\hline \multicolumn{6}{|l|}{ Insolation (h) } \\
\hline 1979 & 127 & 199 & 236 & 280 & 169 \\
\hline 1980 & 156 & 186 & 162 & 175 & 217 \\
\hline 1981 & 162 & 146 & 193 & 193 & 231 \\
\hline Moyenne $1971 / 1980$ & 146 & 171 & 201 & 236 & 193 \\
\hline
\end{tabular}

La figure 1 permet de suivre l'évolution des différences cumulées (ETM - P), où ETM représente l'évapotranspiration maximale de la culture, mesurée chaque semaine sur un dispositif expérimental voisin

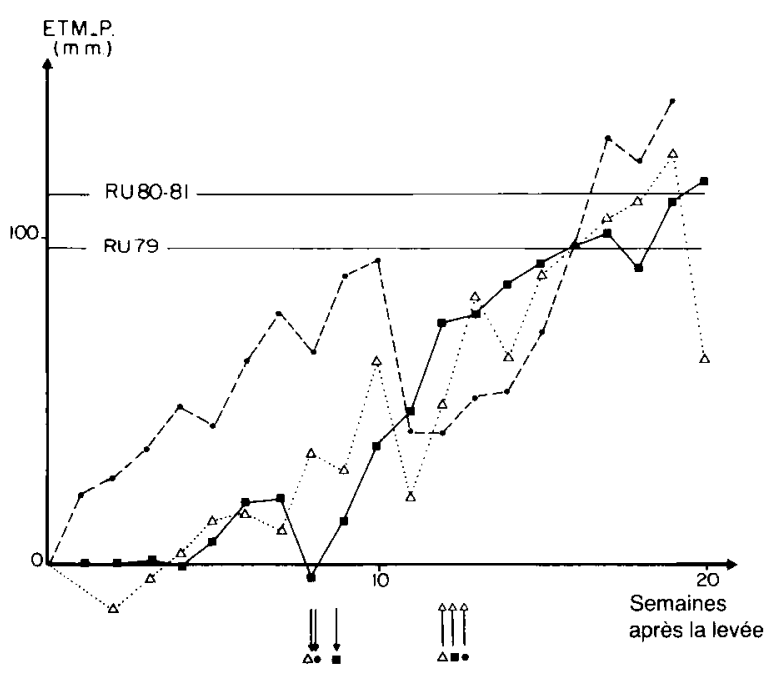

Figure 1

Evolution de (ETM-P) cumulée au cours du cycle.

$E T M=$ évapotranspiration maximale, $P=$ précipitation + irrigation, $R U=$ réserve utile en eau du sol.

- 1979, -1980, $\triangle 1981$ | début floraison 1 fin floraison

Cumulated (ETM-P) value during the vegetative cycle.

ETM = maximal evapotranspiration, $P=$ rain + irrigation, $R U=$ available moisture capacity of the soil

- 1979, - 1980, $\triangle 1981$ | first flowering | end of flowering
(DUTHION \& DUREY, 1984), et P les précipitations et irrigation (apport complémentaire de $65 \mathrm{~mm}$ en 1980 au début floraison des axes A2). Ces différences cumulées dépassent la valeur de la réserve utile en eau du sol, estimée à $97 \mathrm{~mm}$ en 1979 et $113 \mathrm{~mm}$ en 1980 et 1981 , seulement tout en fin de végétation. L'examen de profils, au début floraison des axes A1, a montré en outre qu'à ce stade les systèmes racinaires exploraient l'ensemble du volume de sol à leur disposition. Selon toute vraisemblance, les cultures n'ont pas souffert de la sécheresse pendant la majeure partie du cycle sauf peut-être en 1980 où un déficit hydrique relativement important est apparu au cours de la phase végétative (fig. 1).

\section{B. Prélèvements, mesures et analyses}

Chaque parcelle élémentaire de $16 \mathrm{~m}$ de longueur comprend 6 rangs espacés de $0,30 \mathrm{~m}$. Une moitié en a été réservée pour la récolte mécanique (AMARGER \& DuTHION, 1983). Sur la seconde, des prélèvements de plantes ont été faits en cours de végétation, à intervalles de temps réguliers de 2 semaines à partir des stades 5-6 ou 8-9 feuilles, selon les années, jusqu'à la récolte.

En 1979 , les plantes ont été prélevées sur un mètre linéaire d'une des 4 lignes centrales choisie alternativement, de telle façon que l'environnement des emplacements destinés aux prélèvements suivants soit modifié aussi peu que possible. En 1980 et 1981, 24 plantes ont été prélevées soit 8 plantes consécutives sur 3 des lignes centrales, avec un espacement de $0,5 \mathrm{~m}$ de ligne d'un prélèvement à l'autre.

Les parties aériennes sont seules prélevées. Elles sont séparées en leurs différents organes: tiges, feuilles (limbes + pétioles) de tous ordres d'axes, organes reproducteurs (fleurs, gousses puis cosses et graines) de chaque ordre d'axes en 1979, 1980 ; tiges, feuilles, organes reproducteurs de chaque ordre d'axes en 1981. En 1981, des filets de $50 \mathrm{~cm}$ de longueur, posés sur le sol entre 2 lignes, ont permis de récupérer les organes tombés.

Les échantillons sont séchés à $80^{\circ} \mathrm{C}$ pendant 48 heures puis pesés. Les teneurs en azote total sont déterminées selon la technique de Kjeldahl.

L'évolution de l'activité fixatrice d'azote a été appréciée de manière indirecte par mesure hebdomadaire de l'activité réductrice d'acétylène de racines excisées (AMARGER et al., 1979).

Des déterminations de la quantité d'azote fixé ont été effectuées en 1981, par la méthode isotopique, après léger enrichissement en $15 \mathrm{~N}$ de l'azote minéral ( $5 \mathrm{~kg} \mathrm{~N} /$ ha, sous forme de $\mathrm{KNO}_{3}$, enrichi à 1 p. 100 en $15 \mathrm{~N}$ apporté en pulvérisation au sol). Les mesures ont porté sur les échantillons prélevés à 3 dates différentes. Testée parmi plusieurs espèces, l'avoine, cv Gambo, a été retenue comme plante témoin (AMARGER, résultats non publiés).

\section{RÉSULTATS}

\section{A. Déroulement du cycle végétatif}

REEVES et al. (1977) ont montré que le développement du lupin blanc est sous la dépendance étroite de la température jusqu'au début floraison. Au-delà et 
jusqu'à fin floraison ses besoins en température sont corrélés négativement avec la durée du jour; à l'échelle du territoire français, ils devaient cependant peu varier, surtout si l'on tient compte des décalages des dates de végétation entre sud et nord. La méthode des sommes de températures est alors commode pour caractériser le cycle. Dans nos conditions culturales, les sommes de températures, correspondant à la réalisation de plusieurs stades repères, ont effectivement peu différé d'une année à l'autre. Dans le tableau 2, elles sont exprimées en base $0{ }^{\circ} \mathrm{C}$ et en base $4{ }^{\circ} \mathrm{C}$; l'utilisation de cette dernière, considérée par REEVES et al. (1977) comme le seuil de végétation pour l'espèce et Lupinus angustifolius, n'améliore pas nettement la précision obtenue.

\section{TABLEAU 2}

Sommes de températures pour différents stades repères; moyennes $\left({ }^{\circ} \mathrm{C} . j\right) \pm$ écart-type.

(I) Début floraison = épanouissement de la lre fleur de l'inflorescence pour 50 p. 100 des plantes.

Sum of temperatures for several vegetative stages; means $\left({ }^{\circ} \mathrm{C} . d\right)$ \pm standard deviation.

(1) Flowering $=$ opening of the first flower of inflorescence for $50 \%$ of plants.

base $0{ }^{\circ} \mathrm{C} \quad$ base $4{ }^{\circ} \mathrm{C}$

Sommes de températures depuis la

levée jusqu'à

Stade 5 feuilles

Stade 9-10 feuilles

Début floraison (1) axe principal

Début floraison (1) axes apicaux

Début floraison (1) axes apicaux 2

Début floraison (1) axes apicaux 3

Maturité récolte

$\begin{array}{rr}244 \pm 10 & 137 \pm 19 \\ 407 \pm 24 & 233 \pm 34 \\ 582 \pm 10 & 354 \pm 12 \\ 723 \pm 10 & 460 \pm 7 \\ 897 \pm 22 & 587 \pm 10 \\ 1097 \pm 22 & 735 \pm 10 \\ 2150 \pm 66 & 1554 \pm 51\end{array}$

Développement et croissance sont largement interdépendants (DURAND, 1967). Dans ce qui suit, la représentation du temps en sommes de températures, qui permet de situer l'évolution de la croissance par rapport au développement et facilite les comparaisons interannuelles, a été retenue. La base $0{ }^{\circ} \mathrm{C}$ a été conservée.

\section{B. Production de matière sèche}

L'évolution des quantités totales de MS aérienne au cours du cycle est illustrée par la figure 2 ; il n'y est pas tenu compte de la MS des feuilles et autres organes tombés en cours de végétation, soit $500 \mathrm{~kg} / \mathrm{ha}$ en 1981 .

Sensiblement à la date des premières floraisons, soit 550 à $600^{\circ} \mathrm{C}$-j après la levée, la vitesse d'accumulation de la MS augmente. Durant la période de forte croissance pondérale qui suit, la plante acquiert 85 p. 100 de sa MS, avec des gains moyens de 80 à $110 \mathrm{~kg} / \mathrm{ha} / 10^{\circ} \mathrm{C}-\mathrm{j}$. Les MS totales maximales (considérées telles lorsque les différences avec les MS accumulées à la date suivante ne sont plus significatives au seuil 5 p. 100) sont atteintes à $1600^{\circ} \mathrm{C}$-j environ; elles sont voisines de $10 \mathrm{t} / \mathrm{ha}$. Les décroissances observées en fin de cycle ne sont pas significatives en 1979 et 1980.

Le poids de l'appareil végétatif est maximal vers $1200{ }^{\circ} \mathrm{C}-\mathrm{j}$, date à laquelle prend fin la floraison des axes A3, derniers axes émis ; il décroît ensuite (fig. 3).

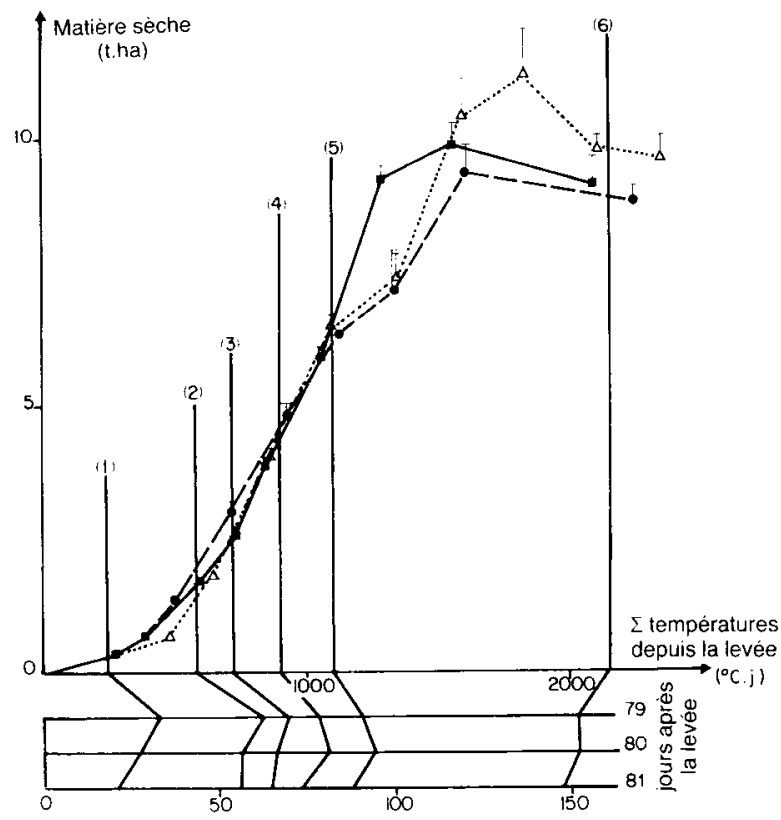

Figure 2

Production de MS aérienne ( $t /$ ha) en fonction des sommes de températures $\left({ }^{\circ} \mathrm{C} . j\right.$; base $\left.0^{\circ} \mathrm{C}\right)$. Les demi-droites verticales indiquent quelques stades repères (sommes de températures moyennes des 3 années): (1) 5 feuilles ou début allongement tige, (2) floraison $A 0$, (3) floraison A1, (4) floraison A2, (5) floraison A3, (6) maturité récolte. La correspondance en nombre de jours réels est donnée pour les 3 années. Les segments verticaux représentent les écarts-types des moyennes.

\section{- 1979, - 1980, $\triangle 1981$}

Aerial dry matter production $(t / h a)$. The $X$ axis represents the sum of temperatures $\left({ }^{\circ} \mathrm{C} . d\right.$; base $\left.O{ }^{\circ} \mathrm{C}\right)$. Vertical half-lines indicate stages (means of the sums of temperatures for the 3 years): (1) 5 leaves or beginning of stem elongation, (2) AO flowering, (3) A1 flowering, (4) A2 flowering, (5) A3 flowering, (6) maturity. Corresponding numbers of days are given for the 3 years. Vertical bars are standard errors of means.

- 1979, $\bullet 1980, \triangle 1981$

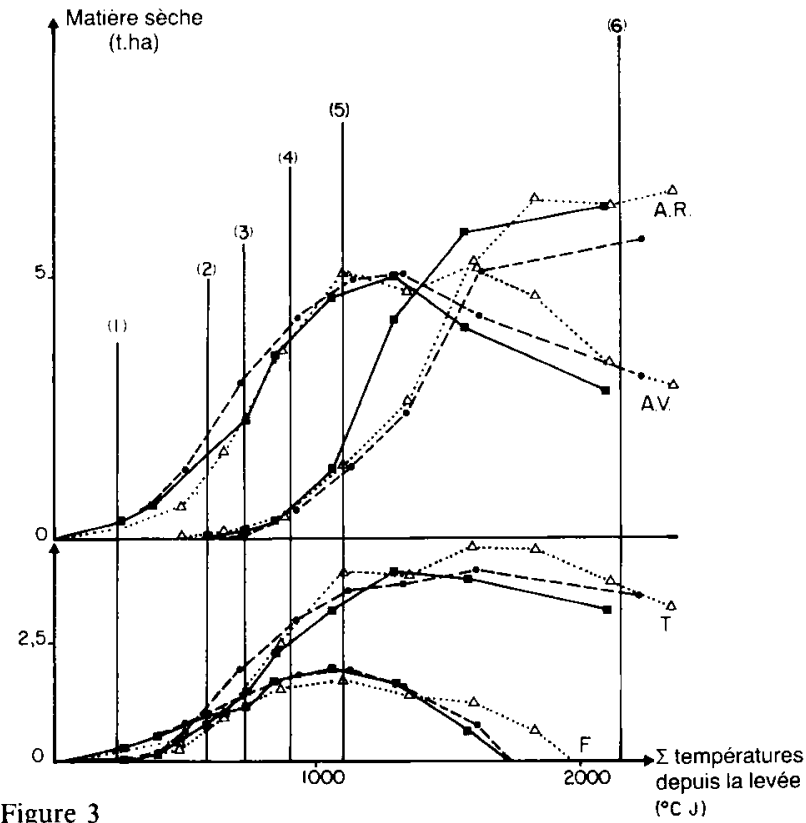

Figure 3

Evolution de la quantité de MS dans différents organes (t/ha). A.R. : appareil reproducteur, A.V. : appareil végétatif, $T:$ tiges, $F:$ feuilles.

Pour la signification des repères et des figurés, voir la figure 2.

Dry matter development for some organs $(t / h a)$.

A.R. : reproductive plant parts, A.V.: vegetative plant parts, $T:$ stems, $F:$ leaves.

For conventions, see figure 2. 
La valeur maximale de la MS foliaire est atteinte plus tôt, vers $1000{ }^{\circ} \mathrm{C}-\mathrm{j}$, bien que l'émission et la croissance de feuilles nouvelles se poursuivent jusqu'à la fin d'allongement des axes de l'ordre le plus élevé ; à ce moment, en effet, les feuilles les plus basses de l'axe $\mathrm{A} 0$ sont déjà tombées. La chute des feuilles, après perte partielle de leur MS, progresse ensuite le long de A0 puis des axes supérieurs ; elle est totale en fin de cycle. Le poids de l'ensemble des tiges augmente jusqu'à $1200-1300{ }^{\circ} \mathrm{C}-\mathrm{j}$; l'allongement d'un axe cesse lorsque débute la floraison de son inflorescence, mais sa croissance pondérale continue par formation de tissus secondaires (FARRINGTON \& PATE, 1981).

L'appareil végétatif accumule une grande part de sa MS, les $2 / 3$ environ dans ces essais, durant la floraison. L'appareil reproducteur s'établit pendant ce temps mais acquiert peu de MS ; dans la première phase de son évolution, le fruit du lupin blanc commence par s'allonger, surtout par rétention d'une grande quantité d'eau (PATE et al., 1977). A partir de la fin floraison, tous les assimilats élaborés et les composés mobilisés aux dépens des organes sénescents sont par contre drainés vers l'appareil reproducteur. Celuici atteint son poids maximal à $1700-1800{ }^{\circ} \mathrm{C}-\mathrm{j}$ environ ; mais à l'intérieur des gousses, les redistributions depuis les cosses vers les graines se poursuivent tardivement (fig. 4).

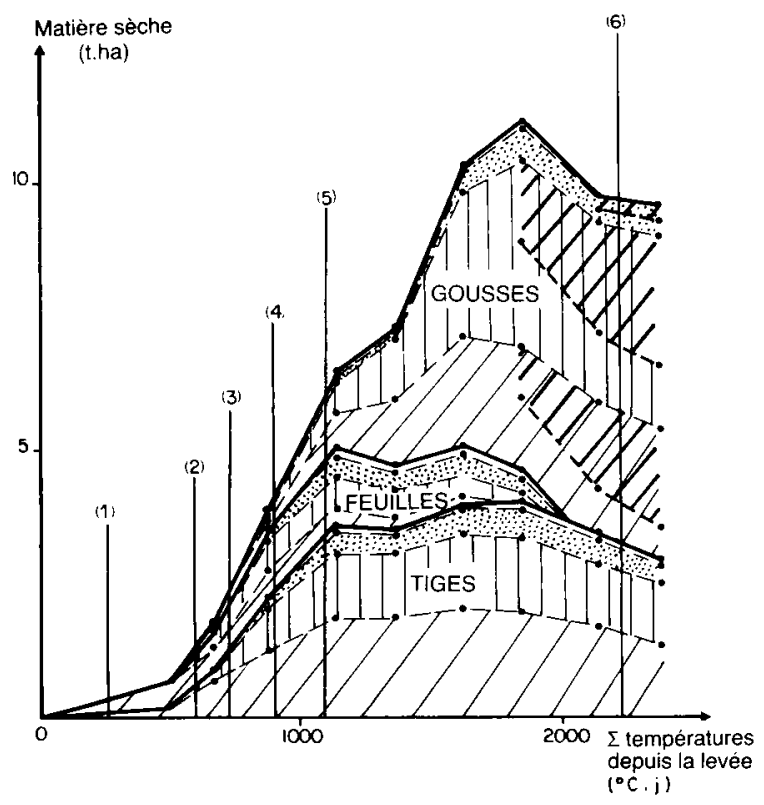

Figure 4

Répartition de la MS aérienne entre les différents organes et les différents niveaux d'axes, année $1981(t / h a)$.

$\square]$ axe $A 0,[10$ axe $A 1, \square$ axe $A 2, \square$ axe $A 3, \square \square$ grains.

Dry matter distribution among the different aerial plant parts, for different axis levels, year 1981 ( $t / h a)$.

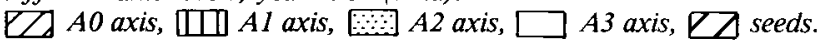
For other conventions, see figure 2.

Aux densités de peuplement réalisées, les axes A0 et A1 constituent les éléments dominants de la structure de la plante; l'exemple de la distribution de la MS entre niveaux d'axes observée en 1981 le montre clairement (fig. 4). Cependant les axes A2 et A3 permettent le maintien d'une activité photosynthétique après sénescence des appareils foliaires des axes inférieurs.
Les organes portés par A2 et A3 contribuent peu au poids de l'appareil reproducteur.

Globalement, les quantités maximales de MS végétative produites par unité de surface ne diffèrent pas entre années, malgré quelques variations de la structure de la plante moyenne, résultant de l'inégalité des densités de peuplement. Le poids maximal de l'appareil reproducteur en 1980 est en revanche significativement inférieur à celui des 2 autres années.

\section{Accumulation d'azote}

L'évolution de l'accumulation totale d'azote dans les parties aériennes des lupins présente une grande similitude avec celle de leur croissance pondérale (fig. 5). On observe une phase de forte accumulation qui débute à la floraison et s'achève vers $1600^{\circ} \mathrm{C}-\mathrm{j}$; les gains moyens pendant cette période sont de $2,5 \mathrm{~kg} \mathrm{~N} / \mathrm{ha} / 10{ }^{\circ} \mathrm{C}-\mathrm{j}$. Les mobilisations avoisinent $300 \mathrm{~kg} \mathrm{~N} / \mathrm{ha}$.

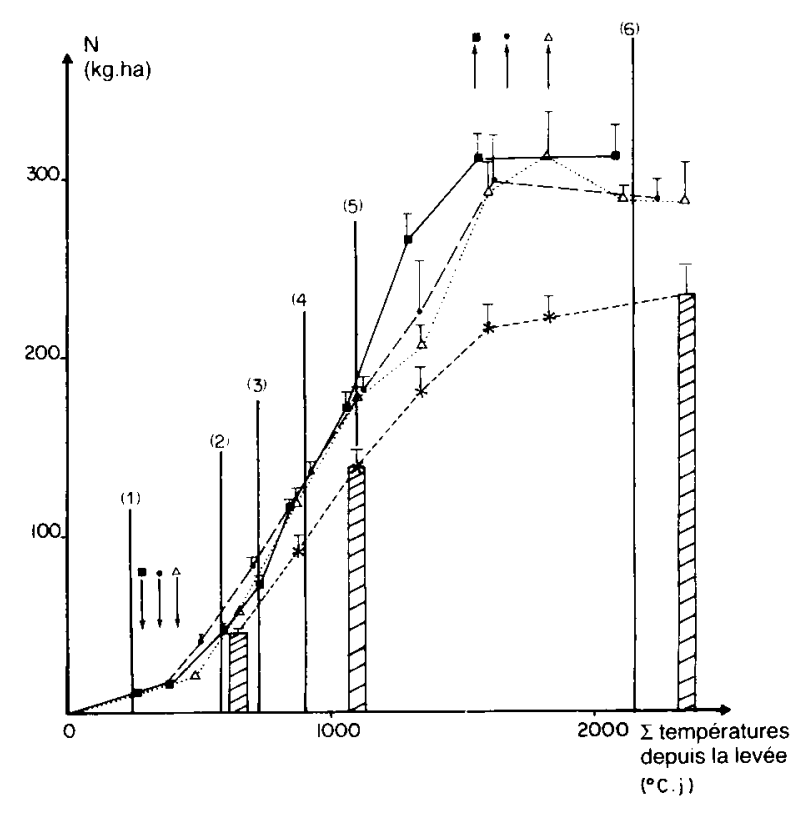

Figure 5

Accumulation d'azote dans les parties aériennes ( $\mathrm{kg} / \mathrm{ha})$.

Les quantités d'azote fixé, mesurées ([ך]) ou estimées (*) d'après AMARGER (résultats non publiés) correspondent à l'année 1981. 1 Début fixation, 1 fin fixation. Pour la signification des repères et des autres figurés, voir la figure 2 . Les segments verticaux représentent les écarts-types de moyennes.

Nitrogen accumulation in aerial plants part $(\mathrm{kg} / \mathrm{ha})$.

Amounts of symbiotically fixed nitrogen, measured by N1S technique (ЕД) or estimated (*) according to AMARGER (non-published results) are 1981 values. I Fixation begins, 1 fixation ends. For other conventions, see figure 2. Vertical bars are standard errors of means.

Le contenu en $\mathrm{N}$ des organes végétatifs est maximal à une date proche de celle à laquelle leur poids est luimême maximal (fig. 3 et 6) ; deux tiers en sont acquis pendant la floraison. L'azote végétatif décroît par la suite fortement, feuilles et tiges s'appauvrissant. L'accumulation de $\mathrm{N}$ par les organes reproducteurs, d'abord parallèle à leur croissance pondérale, est plus importante en valeur relative, en fin de cycle, que celle de leur MS.

La répartition entre organes de la MS et celle de $\mathrm{N}$ 


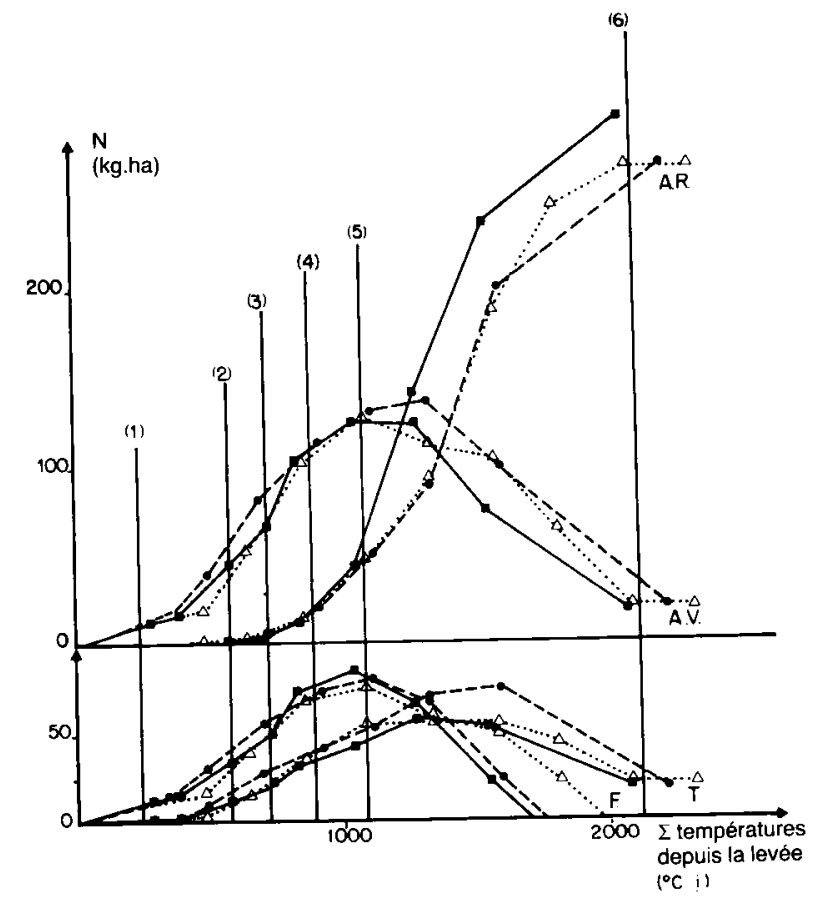

Figure 6

Evolution de la quantité de $N$ accumulé dans différents organes $(t / h a)$. Pour la signification des repères et figurés, voir la figure 3.

Evolution of accumulated $N$ for some organs. For conventions, see figure 3.

diffèrent beaucoup. Pour un même type d'organes végétatifs, on observe également une différence de répartition, leur teneur en $\mathrm{N}$ croissant avec le numéro d'ordre des axes (fig. 4 et 7 ).

Les quantités maximales de $\mathrm{N}$ accumulé par unité de surface au total aussi bien que dans les appareils végé-

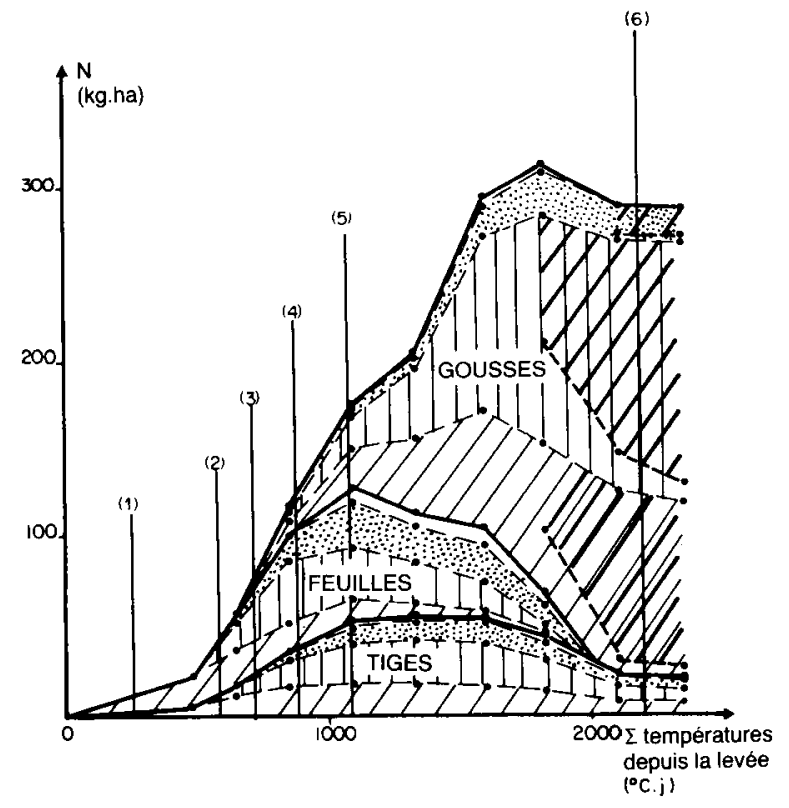

Figure 7

Répartition de $N$ accumulé entre les différents organes et les différents niveaux d'axes, année $1981(\mathrm{~kg} / \mathrm{ha})$. Pour la signification des repères et figurés, voir la figure 4.

Accumulated $N$ distribution among the different aerial plant parts, for the different axis levels, year $1981(\mathrm{~kg} / \mathrm{ha})$. Same conventions as in figure 4. tatif et reproducteur ne diffèrent pas significativement d'une année à l'autre.

Le début de la fixation d'azote atmosphérique, dont on admet la concordance avec la date des $1^{\text {res }}$ mesures significatives de l'activité réductrice d'acétylène des racines, précède immédiatement la phase de pleine croissance (fig. 5). Se maintenant à son niveau maximal, malgré quelques fluctuations, pendant toute la durée de la floraison, elle prend fin peu avant la complète sénescence de l'appareil foliaire. Pour chacun des prélèvements de 1981 pour lesquels elle a été mesurée, la quantité de $\mathrm{N}$ fixé est égale à 80 p. 100 de $\mathrm{N}$ accumulé, atteignant $234 \mathrm{~kg} / \mathrm{ha}$ en fin de végétation ; pour les prélèvements intermédiaires, cette quantité a été évaluée à l'aide de la relation mise en évidence par AMARGER (résultats non publiés) entre azote fixé et somme des activités réductrices d'acétylène (fig. 5). Dans les conditions des essais, fin d'accumulation apparente de $\mathrm{N}$ et fin de fixation symbiotique sont contemporaines.

Le stade limite d'avortement (PIGEAIRE et al., 1986) marque le début du remplissage de la graine des légumineuses. Chez le lupin blanc, ce stade est franchi lorsque le poids sec de la graine dépasse $30 \mathrm{mg}$, poids atteint selon DAviES \& WILLIAMS (1983) 30 à 40 jours après l'anthèse. Le contenu en $\mathrm{N}$ des graines provient du $\mathrm{N}$ incorporé par la plante au cours de la phase de remplissage et de la redistribution partielle pendant cette même phase du $\mathrm{N}$ accumulé précédemment dans l'appareil végétatif et les gousses (SINCLAIR \& DE WIT, 1976). La part de ces 2 processus peut être estimée à partir des variations de contenu en $\mathrm{N}$ des différents compartiments végétaux entre le prélèvement le plus proche de la date vraisemblable de franchissement du stade limite d'avortement par la plante entière (compte tenu de la position de ses graines à maturité) et la récolte. En 1981, année pour laquelle les éléments de bilan sont les mieux connus, les graines à la récolte contiennent $247 \mathrm{~kg} / \mathrm{ha}$ de $\mathrm{N} ; 131 \mathrm{~kg} / \mathrm{ha}$, dont 102 d'origine symbiotique ( $\mathrm{N}$ fixé étant supposé uniformément distribué dans la plante), proviendraient de redistributions et 116 , dont 98 d'origine symbiotique, d'incorporations pendant la phase de remplissage.

Les feuilles sénescentes ont entraîné sur le sol $11 \mathrm{~kg}$ $\mathrm{N} / \mathrm{ha}$ en 1981 ; à moins de pertes telles que transferts vers les racines ou lessivage, 88 p. 100 de leur contenu en $\mathrm{N}$ ont été effectivement redistribués vers les organes reproducteurs. Les tiges, dans leur ensemble, ont perdu au bénéfice de ces derniers 65 p. 100 de leur N. En fin de cycle, les transferts se poursuivent tardivement depuis les cosses des gousses jusqu'aux graines (fig. 7).

\section{Résultats culturaux}

Le rendement machine, ses composantes nombre et poids des graines et la composition de celles-ci sont donnés dans le tableau 3. Le rendement obtenu en 1980 est significativement inférieur à celui des 2 autres années ; par rapport à 1981 un déficit de production des axes A1 est observé. La comparaison 1979-1981 montre que, pour une même production totale, la participation au rendement des différents ordres d'axes dépend de la densité de peuplement. La teneur en $\mathbf{N}$ 


\section{TABLEAU 3}

Résultats culturaux (sur une ligne, les nombres suivis de la même lettre ne sont pas significativement différents au seuil 5 p. 100).

Yields and seed contents (numbers in a line which are followed by the same letter are not significantly different at the $5 \%$ level).

\begin{tabular}{|c|c|c|c|}
\hline & 1979 & 1980 & $198 I$ \\
\hline $\begin{array}{l}\text { Rendements graines } \\
\left(\mathrm{t} / \mathrm{ha} \text { à } 0 \text { p. } 100 \mathrm{H}_{2} \mathrm{O}\right)\end{array}$ & $4,1 \mathrm{~b}$ & 3,3 a & $4,2 \mathrm{~b}$ \\
\hline $\begin{array}{c}\text { dont sur axe A0 } \\
\text { sur axes A1 } \\
\text { sur axes A2 }\end{array}$ & $\begin{array}{r}2,4 \mathrm{~b} \\
1,7 \mathrm{~b} \\
<0,1 \mathrm{a}\end{array}$ & $\begin{array}{l}1,7 \mathrm{a} \\
1,4 \mathrm{a} \\
0,2 \mathrm{~b}\end{array}$ & $\begin{array}{l}1,6 \mathrm{a} \\
2,3 \mathrm{c} \\
0,3 \mathrm{c}\end{array}$ \\
\hline $\begin{array}{l}\text { Poids } 1000 \text { graines } \\
\left(\mathrm{g} \text { à } 0 \text { p. } 100 \mathrm{H}_{2} \mathrm{O}\right)\end{array}$ & 244 & 247 & 243 \\
\hline Nombre graines récoltées $/ \mathrm{m}^{2}$ & $1677 \quad b$ & 1337 a & a $1715 \quad b$ \\
\hline $\mathrm{N}$ graines (p. $100 \mathrm{MS})$ & $6,3 \mathrm{~b}$ & $6,7 \mathrm{c}$ & $5,9 \mathrm{a}$ \\
\hline $\begin{array}{l}\text { Matières grasses graines } \\
\text { (p. } 100 \mathrm{MS} \text { ) }\end{array}$ & $8,6 \mathrm{a}$ & $8,4 \mathrm{a}$ & $8,7 \mathrm{a}$ \\
\hline
\end{tabular}

des graines diffère significativement d'une année à l'autre.

Pour produire $100 \mathrm{~kg}$ de graines, l'accumulation d'environ 7,5 kg de $\mathrm{N}$ a été nécessaire en 1979 et 1981 ; elle a été de $9 \mathrm{~kg}$ en 1980.

Les indices de récolte pour la MS, calculés à partir des prélèvements de plantes faits à maturité, sont égaux à $0,49,0,44$ et 0,46 en 1979,1980 et 1981 (indice de 1979 significativement supérieur) ; les productions en graines évaluées à partir de ces prélèvements sont respectivement supérieures de 8,12 et 5 p. 100 à celles qui ont été déterminées par récolte mécanique. Les indices de récolte pour l'azote ont pour valeur successivement $0,90,0,88$ et 0,86 (différence significative entre les valeurs de 1979 et 1981).

\section{DISCUSSION}

Dans les conditions de ces essais, les dates qui marquent les étapes successives de l'évolution de la MS totale et celle du $\mathrm{N}$ total des parties aériennes diffèrent peu ; en alimentation azotée uniquement minérale, HOCKING \& PATE (1977) avaient noté en revanche que l'accumulation de $\mathrm{N}$ est plus précoce que l'élaboration de MS. Dans la plante, MS et N ne sont pas distribués de la même façon; pour un type d'organes donné, poids sec et contenu en $\mathbf{N}$ ne suivent pas toujours des évolutions parallèles, surtout en fin de cycle.

Production de MS et accumulation de $\mathrm{N}$ ont été du même ordre de grandeur au cours des 3 années expérimentales ; les différences de peuplement ont été compensées par une croissance individuelle plus importante dans le cas des densités les plus faibles. Le rendement inférieur obtenu en 1980 est dû à un nombre moindre de graines produites. La cause la plus probable en est l'attaque par $B$. cinerea ( $\S$ II A1), qui a provoqué des dommages surtout sur gousses A1 alors en cours de croissance; d'autres facteurs, auxquels il faudrait cependant attribuer des effets spécifiques sur la fructification, pourraient être aussi invoqués, telles des tem- pératures et insolations inférieures aux moyennes régionales pendant une partie du cycle ou une moindre disponibilité temporaire en eau (fig. 1). Le rapport des quantités de $\mathrm{N}$ potentiellement disponibles pour une redistribution à partir des organes végétatifs au nombre de graines à remplir pourrait expliquer la richesse relative en $\mathbf{N}$ de la récolte de cette même année.

Les mobilisations vers les fruits du carbone assimilé pendant la croissance végétative sont faibles (PATE \& Minchin, 1978). PATE et al. (1980) ont montré que 95 p. $100 \mathrm{du}$ carbone des fruits de L. angustifolius proviennent de l'activité photosynthétique après l'anthèse. Les éléments de la structure de la plante sont en effet d'une grande stabilité. Les réserves en $\mathrm{N}$ sont en revanche beaucoup plus accessibles. Les mobilisations à partir du $\mathrm{N}$ accumulé dans l'appareil végétatif et les enveloppes des gousses avant la phase de remplissage correspondent à $53 \mathrm{p} .100$ de l'azote des graines en 1981. Dans un essai en conditions contrôlées, PATE et al. (1981) ont obtenu des proportions voisines, 57 et 43 p. 100 du $\mathrm{N}$ des graines venant respectivement de redistributions et d'accumulations nouvelles pendant la phase de remplissage. L'indice de récolte observé pour la MS est de 0,40 environ, rapporté au rendement récolte mécanique ; il est de 0,80 pour l'azote.

Selon nos observations, l'activité fixatrice de $\mathrm{N}$ s'exerce chez le lupin blanc pendant une grande partie de son cycle. DENISON \& SinClaIR (1985) constatent que la fixation chez le soja se prolonge aussi jusqu'aux derniers stades de la croissance des graines, lorsque la plante entière est sénescente ; ils soulignent que beaucoup d'observations faites par d'autres auteurs, en conditions d'environnement non contraignantes, confirment ce fait. En 1981, la fixation a assuré 80 p. 100 de la fourniture de $\mathrm{N}$ à la plante, représentant $234 \mathrm{~kg} / \mathrm{ha}$ en fin de végétation. Cette quantité est élevée par rapport à la plupart des données que LARRUE \& PATTERSON (1981) ont recensées pour les différentes espèces de légumineuses à graines ; beaucoup parmi ces données ont cependant été obtenues par la méthode dite des différences (WILliaMs et al., 1977), qui tend à les sous-estimer (AMARGER et al., 1979).

Les rendements que ces essais ont permis en 1979 et 1981 se placent au niveau des meilleurs qui aient été observés. En 10 années sur le domaine expérimental de Bretenière, le rendement du cv Kali a dépassé nettement $4 \mathrm{t} / \mathrm{ha}$ de graines en une seule occasion, atteignant $4,8 \mathrm{t} / \mathrm{ha}$ en 1978 dans un essai irrigation avec conduite à l'ETM, résultat qui n'a pas été reproduit par la suite. Dans l'espèce, parmi les types printemps de la même précocité, convenant à la plupart des régions, le cv Lublanc est à présent le plus utilisé ; ses meilleurs rendements sont aussi de l'ordre de $4 \mathrm{t} / \mathrm{ha}$, avec un maximum de $5 \mathrm{t}$ obtenu en 1981 dans le Centre-Ouest (LENOBLE, communication personnelle). Les résultats présentés donnent donc une bonne image du potentiel de croissance des cv actuels.

Pour l'espèce, HILL (1977) indique, pour limites de la teneur en protéines des graines, les valeurs de 35 et 45 p. 100 de la MS, soit 5,5 et 7,2 p. 100 en N. La plupart des concentrations mesurées pour les $\mathrm{cv}$ et lignées, qui ont été étudiées sur le domaine expérimental de Bretenière, sont, avec des variations interannuelles, effectivement comprises entre 5,5 et 6,5 p. 100 de la MS. Les teneurs observées ici se placent bien dans la partie haute de cette gamme. 
En bonnes conditions d'alimentation hydrique et azotée, le cv Kali a donc élaboré environ $10 \mathrm{t} / \mathrm{ha}$ de MS, pour produire $4 \mathrm{t}$ de graines, et accumulé $300 \mathrm{~kg}$ $\mathrm{N} /$ ha ; compte tenu de ce qui précède, ces quantités peuvent être considérées comme des objectifs de production pour la culture du lupin blanc de printemps en France.

Comparer ces données aux résultats obtenus avec le soja, dont le lupin blanc est un substitut potentiel, permet de situer mieux encore les possibilités de l'espèce. Les travaux de HANWAY \& WEBER $(1971 a, b, c)$ sont souvent cités comme références pour le soja. Les vitesses de croissance pondérale, d'accumulation de $\mathrm{N}$ que nous avons mesurées sont voisines de celles que ces auteurs ont obtenues chez des cv de soja du groupe II de précocité ; dans leurs essais le cv Hawkeye a élaboré $10 \mathrm{t} /$ ha de MS, comprenant les feuilles tombées, pour une récolte de $3 \mathrm{t} /$ ha de graines, et accumulé $270 \mathrm{~kg} / \mathrm{ha}$ de $\mathrm{N}$ dont $70 \mathrm{p} .100$ sont dans les graines à maturité. Beaucoup d'auteurs américains fournissent des résultats du même ordre de grandeur (EGLı \& LEGGETT, 1973 ; BEAVER et al., 1985). SHIBLES et al. (1975) admettent des rendements fréquents en culture de $3 \mathrm{t} /$ ha, pouvant atteindre $4 \mathrm{t} /$ ha occasionnellement. En France, PUECH et al. (1974) ont obtenu jusqu'à $10,3 \mathrm{t} /$ ha de MS totale et $4 \mathrm{t} / \mathrm{ha}$ de graines en conditions favorables avec le cv Amsoy du groupe II de précocité. Dans un document récent, PUECH \& BoUNIOLS (1986) fournissent des données très voisines, avec des mobilisations en $\mathrm{N}$ de l'ordre de $300 \mathrm{~kg}$ et PLANCHON et al. (1986), à partir d'un essail portant sur des cv de précocité 00 à III, montrent qu'en l'absence de contrainte hydrique le rendement maximal, dans ce cas un peu supérieur à $4 \mathrm{t} / \mathrm{ha}$, est obtenu dans le Sud-Ouest avec les groupes I et II. Pour des sojas de fort rendement, l'assimilation nette du couvert, calculée par BLANCHET et al. (1982) serait de 24 à $27 \mathrm{t} /$ ha de $\mathrm{CO}_{2}$ sur la totalité du cycle ; selon la même méthode d'estimation, fondée sur les travaux de PENNING DE VRIES (1975), le lupin blanc dans nos essais aurait assimilé $24 \mathrm{t} /$ ha de $\mathrm{CO}_{2}$ en 1981 , dont les graines à maturité représentent l'équivalent de 11,6 t.

\section{CONCLUSION}

Obtenus en l'absence de contraintes hydriques ou azotées marquées, les résultats commentés dans cet article fournissent une bonne approximation des potentiels d'accumulation de MS et $\mathrm{N}$ et de rendement des cv précoces actuels du lupin blanc, en culture de printemps. Ces $\mathrm{cv}$ sont capables d'élaborer $10 \mathrm{t} / \mathrm{ha} \mathrm{de}$ MS, pour une production de $4 \mathrm{t}$ de graines, et d'accumuler $300 \mathrm{~kg} /$ ha de $\mathrm{N}$.

Dans nos conditions, le développement des plantes est limité à l'émission des axes A3 ; mais dans les peuplements denses réalisés la production des graines est assurée par $\mathrm{A} 0$ et $\mathrm{A} 1$, dans des proportions respectives variables avec la densité et les années.

Les évolutions de la MS et de N présentent des similitudes ; en particulier les périodes de pleine croissance et de forte accumulation de $\mathrm{N}$ coïncident sensiblement. Durant la mise en place de l'appareil reproducteur, les organes végétatifs exercent une forte attraction envers les assimilats et l'azote ; deux tiers de leur MS et leur $\mathrm{N}$ sont acquis pendant la floraison. Au-delà de cette période tous les assimilats élaborés et les produits mobilisés aux dépens des organes végétatifs sénescents sont dirigés vers les organes reproducteurs; plus de 50 p. 100 du $\mathrm{N}$ des graines proviennent de redistributions à l'intérieur de la plante.

Les besoins en azote pour produire $0,1 \mathrm{t}$ de graines ont été en moyenne de $8 \mathrm{~kg}$. Environ 80 p. 100 de l'azote accumulé ont été d'origine symbiotique ; l'activité fixatrice, débutant peu avant la floraison, s'est poursuivie pendant une grande partie du cycle, jusqu'à sénescence presque complète de l'appareil foliaire.

Les résultats culturaux que l'on peut attendre du lupin blanc sont proches de ceux que l'on connaît pour le soja. Son rendement potentiel est sans doute un peu plus élevé mais sa graine est moins riche en protéines et matières grasses; en bonnes conditions culturales les assimilations nettes de couverts des 2 espèces sont voisines.

Reçu le 17 décembre 1986. Accepté le 27 avril 1987.

\section{RÉFÉRENCES BIBLIOGRAPHIQUES}

\begin{abstract}
Amarger N., Mariotti A., Mariotti F., Dure J. C., Bourguignon C., Lagacherie B., 1979. Estimate of symbiotically fixed nitrogen in field-grown soybeans using variations in $15 \mathrm{~N}$ natural abundance. Plant Soil, 52, 269-280.

Amarger N., Duthion C., 1983. Effets de l'inoculation par Rhizobium et de la fertilisation azotée sur les rendements et la teneur en azote des graines de lupin (Lupinus albus, L. mutabilis, L. luteus). Agronomie, 3, 995-1000.
\end{abstract}

Beaver J. S., Cooper R. L., Martin R. J., 1985. Dry matter accumulation and seed yield of determinate and indeterminate soybeans. Agron. J., 77, 675-679.

Berthaut J., Sixdenier G., 1978. Les lupins. Mise au point et premiers résultats sur les cultures de printemps, p. 343-346. In Nouvelles des Fourrages à l'I.N.R.A. S.E.I., Versailles, 389 p.

Blanchet R., Merrien A., Gelfi N., Cavalié G., Courtiade B., Puech J., 1982. Evolution et estimation comparée de l'assimilation nette de couverts de mais (Zea mays L.), tournesol (Helianthus annuus L.) et soja (Glycine max (L.) Merrill), au cours de leurs cycles de développement. Agronomie, 2, 149-154.
Davies S., Williams W., 1983. Rates of pod and seed development in Lupinus albus, L. mutabilis, Vicia faba, Pisum sativum and Lathyrus latifolius. In $\mathrm{R}$. Thompson \& R. Casey. Perspectives for peas and lupins as protein crops, 87-93. Martinus Nijhoff Publishers, La Haye.

Denison R. F., Sinclair T. R., 1985. Diurnal and seasonal variation in dinitrogen fixation (acetylene reduction) rates by field-grown soybeans. Agron. J., 77, 679-684.

Durand R., 1967. Action de la température et du rayonnement sur la croissance. Ann. Phys. vég., 9, 5-27.

Duthion C., Durey G., 1984. Quelques données à propos de l'alimentation hydrique du lupin blanc, p. 141-147. In Pourquoi Pois... ? Journée nationale sur les Protéagineux. ITCF, Paris, $311 \mathrm{p}$.

Egli D. B., Leggett J. E., 1973. Dry matter accumulation patterns in determinate and indeterminate soybeans. Crop Sci., 13, 220-222.

Farrington P., Greenwood E. A. N., 1975. Description and specification of the branching structure of lupins. Aust. J, agric. Res., 26, 507-510. 
Farrington P., Pate J. S., 1981. Fruit set in Lupinus angustifolius cv. Unicrop. I. Phenology and growth during flowering and early fruiting. Aust. J. Plant Physiol., 8, 293-305.

Gataulina G. G., Berezhnaya Z. G., 1971. Dynamics of nutrient consumption by plants of white lupin (en russe). Izv. Timiryazevsk. S-kh. Akad., 57-65 (In : Soils Fertil., 1972, 35, 517).

Gladstones J. S., 1970. Lupins as crop plants. Field Crop Abstr., 23, 123-148.

Hanway J. J., Weber C. R., 1971a. Dry matter accumulation in eight soybean (Glycine max (L.) Merrill) varieties. Agron. J., 63, 227-230.

Hanway J. J., Weber C. R., 1971b. Dry matter accumulation in soybean (Glycine $\max (\mathrm{L}$.) Merrill) plants as influenced by N, P and $\mathrm{K}$ fertilization. Agron. J., 63, 263-266.

Hanway J. J., Weber C. R., 1971c. Accumulation of N, P a' ' $\mathrm{K}$ by soybean (Glycine max (L.) Merrill) plants. Agron. J., 63,

Hill G. D., 1977. The composition and nutritive value of lup 1 . Nutr. Abstr. Rev., B, 47, 511-529.

Hocking P. J., Pate J. S., 1977. Mobilization of minerals to developing seeds of legumes. Ann. Bot., 41, 1259-1278.

Laconde J. P., 1985. Aspects techniques de la culture du lupin de printemps. Fr, agric., 1, 39-40.

Larrue T. A., Patterson T. G., 1981. How much nitrogen do legumes fix. In N. C. Brady, Advances in Agronomy, 15-38. Academic Press, New York, 34, 485 p.

Lenoble M., 1984. Sélection des lupins, p. 62-64. In Pourquoi Pois... ? Journée nationale sur les Protéagineux. ITCF, Paris, $311 \mathrm{p}$

Pate J. S., Minchin F. R., 1978. Comparative studies of carbon and nitrogen nutrition of selected grain legumes. In R. J. Summerfield, A. H. Bunting, Advances in Legume science, 105-114. Royal Botanic Garden, Kew.

Pate J. S., Sharkey P. J., Atkins C. A., 1977. Nutrition of a developing legume fruit. Plant Physiol., 59, 506-510.

Pate J. S., Atkins C. A., Perry M. W., 1980. Significance of photosynthate produced at different stages of growth as carbon source for fruit filling and seed reserve accumulation in Lupinus angustifolius L.. Aust. J. Plant Physiol., 7, 283-297.
Pate J. S., Atkins C. A., Herridge D. F., Layzell D. B., 1981. Synthesis, storage and utilization of amino compounds in white lupine (Lupinus albus L.). Plant Physiol., 67, 37-42.

Penning de Vries F. W. T., 1975. Use of assimilate in higher plants. In J. P. Cooper. Photosynthesis and productivity in different environments, 459-461. Cambridge University Press, Cambridge.

Pigeaire A., Duthion C., Turc O., 1986. Characterization of the final stage in seed abortion in indeterminate soybean, white lupin and pea. Agronomie, 6, 371-378.

Planchon C., Calmès J., Blanchet R., 1986. Ecophysiologie du soja. II. Adaptation aux conditions sèches, p. 79-87. In Le soja, physiologie de la plante et adaptation aux conditions francaises. CETIOM, Paris, $182 \mathrm{p}$

Puech J., Bouniols A., 1986. Besoins en eau et en azote du soja : importance des phases sensibles, p. 24-31. In Le soja, physiologie de la plante et adaptation aux conditions françaises. CETIOM, Paris, $182 \mathrm{p}$.

Puech J., Lencrerot P., Hernandez M., 1974. Rôle de quelques facteurs du milieu dans la production quantitative et qualitative du soja. I. Croissance, développement et rendement du soja en culture irriguée ou non. Ann. agron., 25, 659-679.

Reeves T. G., Boundy K. A., Brooke H. D., 1977. Phenological development studies with Lupinus angustifolius and $L$. albus in Victoria. Aust. J. exp. Agric. anim. Husb., 17, 637-644.

Shibes R., Anderson I. C., Gibson A. H., 1975. Soybean. In L. T. Evans. Crop Physiology, 151-189. Cambridge University Press, Cambridge.

Sinclair T. R., de Wit C. T., 1976. Analysis of the carbon and nitrogen limitations to soybean yield. Agron. J., 68, 319-324.

Vavilov V. V., Gataulina G. G., 1982. Development and formation of Lupinus albus yield. In Proceedings II international lupine conference, 166-169. Ministerio Agricultura, Pesca y Alimentacion, Madrid, $409 \mathrm{p}$.

Williams W. A., Jones M. B., Delwiche C. C., 1977. Clover Nfixation measurement by total- $\mathrm{N}$ difference and $15 \mathrm{~N}$ A-values in lysimeters. Agron. J., 69, 1023-1024. 